MATHEMATICS OF COMPUTATION

Volume 72 , Number 241, Pages 247-262

S $0025-5718(02) 01448-5$

Article electronically published on June 4, 2002

\title{
A HOLISTIC FINITE DIFFERENCE APPROACH MODELS LINEAR DYNAMICS CONSISTENTLY
}

\author{
A. J. ROBERTS
}

\begin{abstract}
I prove that a centre manifold approach to creating finite difference models will consistently model linear dynamics as the grid spacing becomes small. Using such tools of dynamical systems theory gives new assurances about the quality of finite difference models under nonlinear and other perturbations on grids with finite spacing. For example, the linear advection-diffusion equation is found to be stably modelled for all advection speeds and all grid spacings. The theorems establish an extremely good form for the artificial internal boundary conditions that need to be introduced to apply centre manifold theory. When numerically solving nonlinear partial differential equations, this approach can be used to systematically derive finite difference models which automatically have excellent characteristics. Their good performance for finite grid spacing implies that fewer grid points may be used and consequently there will be less difficulties with stiff rapidly decaying modes in continuum problems.
\end{abstract}

\section{INTRODUCTION}

Following the introduction of holistic finite differences in [18, 11], we would like to investigate numerical models for the dynamics of a field $u(x, t)$ evolving according to nonlinear reaction-diffusion equations such as $u_{t}=u_{x x}+f\left(u, u_{x}\right)$. This particular class includes Burgers' equation, $f=-u u_{x}$, and autocatalytic reactions, such as $f=u(1-u)$. However, before attacking such nonlinear problems, here we restrict attention to proving that the new methodology accurately models the dynamics of quite general linear PDE's.

Modern dynamical systems theory has had to date very little impact on classical numerical approximations. Indeed, the very first sentence in García-Archilla and Titi [9] says "Finite-element methods seem not to have benefited as much as spectral methods from some of the recent advances in the Dynamical Systems approach to partial differential equations." The concept of inertial manifolds has been developed to capture the long-term, low-dimensional dynamics of dissipative PDE's [20]. However, most efforts to construct approximations to an inertial manifold have been based upon the global nonlinear Galerkin method of Roberts [15], Foias et al. [5] and Marion and Temam [12. This is so even for the variants explored by Jolly et al. [10] and Foias and Titi [8]. In contrast, the approach proposed here is based purely upon the local dynamics on relatively small elements while maintaining, as do inertial manifolds, fidelity with the solutions of the original PDE.

Received by the editor April 6, 2000 and, in revised form, November 14, 2000.

2000 Mathematics Subject Classification. Primary 37L65, 65M20, 37L10, 65P40, 37M99.

(C)2002 American Mathematical Society 
I propose [18] to use centre manifold theory to construct finite difference models. For problems in one spatial dimension I consider implementing the method of lines by discretising in space $x$ and integrating in time $t$ as a set of ordinary differential equations, sometimes called a semi-discrete approximation [6] 8, e.g.]. I only address spatial discretisation and treat the resulting set of ordinary differential equations as a continuous time dynamical system. Classical finite difference approximations are made by appealing to consistency in the limit as the grid spacing $h \rightarrow 0$; traditionally one constructs models to errors $\mathcal{O}\left(h^{2}\right)$ or $\mathcal{O}\left(h^{4}\right)$ depending upon small $h$ asymptotics, shown schematically by the rightward-pointing arrows in Figure 1, In contrast, we here analyse the dynamics at fixed grid spacing $h$ and use centre manifold theory to accurately model the nonlinear dynamics - theory [1, e.g.] assures us that the low-dimensional, numerical model then accurately captures the dynamics in an expansion in the nonlinearity, shown schematically as the forward-pointing arrows in Figure 1. The analysis rests upon the exponential decay of the small, subgrid structures in each local element. Being essentially local in space, the analysis here is flexible enough to subsequently cater for spatial boundaries and spatially varying coefficients (the subject of ongoing research). I call the model "holistic" because the centre manifold is made up of actual solutions of the PDE, thereby accounting for all interactions between physical processes, including subgrid interactions; the model is also consequently invariant under algebraic rewriting of the governing equations. However, to apply the centre manifold theory we have to use a homotopy in a parameter $\gamma$ : when $\gamma=0$ the discrete finite elements of the domain are completely uncoupled from each other; when $\gamma=1$, the requisite continuity is reclaimed and the physical PDE solved. The caveat is that the centre manifold model has to be used at $\gamma=1$ whereas the supporting theory only guarantees accuracy in a neighbourhood of $\gamma=0$; we aim to make the useful neighbourhood big enough to include the relevant $\gamma=1$ (this sort of technique has proven effective in thin fluid flows [16, e.g.]). One way to reasonably secure the centre manifold model, and the way explored herein, is to require that the model is also consistent with the PDE as the grid spacing $h \rightarrow 0$. Thus we aim to construct finite difference numerical models that not only are justified by their asymptotic expansions in nonlinearity and $\gamma$, but are also justified by asymptotics in $h$ (see Figure11). This dual justification is the completely novel feature of the approach.

The first step, taken in this paper, is to establish a centre manifold approach that is also guaranteed to construct a consistent finite difference model for a general linear PDE, shown schematically in Figure 1 as the disc in the $\gamma h$-plane (zero nonlinearity). We leave to later research the problem of guaranteeing the consistent modelling of nonlinear dynamics. Herein we explore the finite difference modelling of the linear PDE

$$
\frac{\partial u}{\partial t}=\mathcal{A} u+\epsilon \mathcal{B} u,
$$

where the linear operator $\mathcal{A}$, presumed generally dissipative, is even (it contains only even order derivatives in $x$ with constant coefficients), and $\mathcal{B}$ is an odd linear operator (it contains only odd order derivatives with constant coefficients); $\mathcal{A}$ is assumed dissipative for all modes except $u=$ const. The case of space-time varying coefficients to the linear problem is also left for later study; however, I expect that because the analysis here is local in $x$, then such varying coefficients can be treated as a perturbing influence to the basic analysis herein. As a specific example, we 


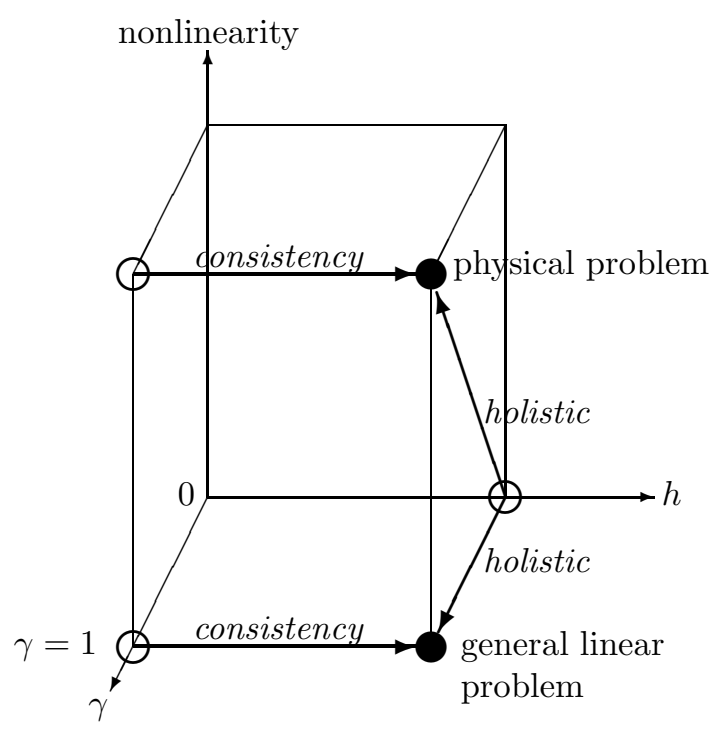

Figure 1. Conceptual diagram showing: the traditional finite difference modelling approaches (rightward-pointing arrows) the physical problem (upper disc) via asymptotic consistency as the grid size $h \rightarrow 0$ (left circles); whereas the holistic method approaches (forward-pointing arrows) the physical problem via asymptotics in nonlinearity and the inter-element coupling $\gamma$ (from right circle). Herein we establish how to use the holistic approach to do both in order to model a general linear problem (lower disc).

discuss in 93 the linear advection-diffusion equation $u_{t}=-\epsilon u_{x}+u_{x x}$ and discover many remarkable properties of the holistic finite difference models. The separation between the two types of linear terms into $\mathcal{A} u$ and $\epsilon \mathcal{B} u$ occurs because first we prove consistency for even terms, 4 , before moving on to prove consistency for the odd terms, 5 .

Introduce a regular grid as shown in Figure 2 with grid points a distance $h$ apart, $x_{j}=j h$ for example, and, using $u_{j}$ to denote the value of the field at each grid point,

$$
u_{j}(t)=u\left(x_{j}, t\right) .
$$

We express the field in the neighbourhood of the $j$ th grid point by $u=v_{j}(x, t)$. We do not restrict the function $v_{j}$ to just the $j$ th element, but allow it to extend analytically out to at least the adjacent grid points as shown in Figure 2

Herein we establish that small $h$ consistency follows from using the nonlocal, internal "boundary conditions"

$$
v_{j}\left(x_{j \pm 1}, t\right)=(1-\gamma) v_{j}\left(x_{j}, t\right)+\gamma v_{j \pm 1}\left(x_{j \pm 1}, t\right) .
$$

That is, the field of the $j$ th element when evaluated at the surrounding gridpoints, $v_{j}\left(x_{j \pm 1}, t\right)$, is a continuation between two critical extremes: when $\gamma=1$, it is the field at those grid points, $v_{j \pm 1}\left(x_{j \pm 1}, t\right)$, to in effect recover the physical continuity as shown schematically in Figure 2 but when $\gamma=0$, the field is just identical to the 


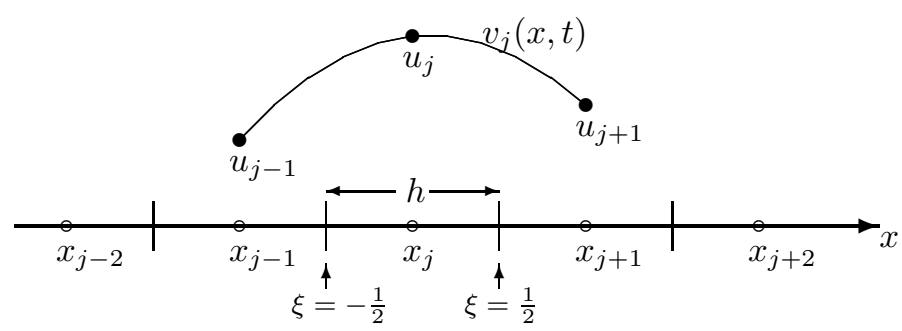

FigURE 2. Schematic picture of the equi-spaced grid, $x_{j}$ spacing $h$, the unknowns $u_{j}$, the artificial internal boundaries between each element (vertical lines), and in the neighbourhood of $x_{j}$ the field $v_{j}(x, t)$ which extends outside the element and, if $\gamma=1$, passes through the neighbouring grid values $u_{j \pm 1}$.

mid-element value $v_{j}\left(x_{j}, t\right)$ so that an element becomes isolated from all neighbours. Equivalently, (3) is transformed to the following appealing two difference equations 1 evaluated at the centre of the element, $x=x_{j}$,

$$
\mu_{x} \delta_{x} v_{j}(x, t)=\gamma \mu \delta v_{j}\left(x_{j}, t\right) \text { and } \delta_{x}^{2} v_{j}(x, t)=\gamma \delta^{2} v_{j}\left(x_{j}, t\right) .
$$

That is, in the two extremes: when $\gamma=0$ the first and second differences have to be zero; whereas when $\gamma=1$ the first two differences of the field centred on each element have to agree with the first two differences of the grid values. Note a distinction which is very important throughout this work: unadorned difference operators, such as the central mean $\mu=\left(E^{1 / 2}+E^{-1 / 2}\right) / 2$ and central difference $\delta=E^{1 / 2}-E^{-1 / 2}$ written in terms of the shift operator $E u_{j}=u_{j+1}$ [13, p. 64, e.g.], apply to the grid index $j$ (with step 1 ) whereas those with subscript $x$, as in $\mu_{x}$ and $\delta_{x}$, are differences in $x$ only (with step $h$ ). Using the definition of the amplitudes (2), these internal boundary conditions (IBC) simplify to the following form which we use throughout $\S \S 3$ 5: evaluated at $x=x_{j}$ :

$$
\mu_{x} \delta_{x} v_{j}(x, t)=\gamma \mu \delta u_{j} \quad \text { and } \quad \delta_{x}^{2} v_{j}(x, t)=\gamma \delta^{2} u_{j} .
$$

In actually developing finite difference models these IBC's may take any of many equivalent forms [18, e.g.]. Small $h$ consistency seems easiest to establish in this particular discrete form.

\section{The Dynamics COllapses onto a CEntRe Manifold}

I establish here the basis of a centre manifold analysis of the linear PDE (1). It appears necessary, and is the route taken here, to separate the linear effects into those generated by even terms, represented by $\mathcal{A}$ and presumed generally dissipative on the grid scale $h$ but with one 0 eigenvalue corresponding to $u=$ const 2 and those generated by odd terms, represented by $\epsilon \mathcal{B}$. The analysis is to be based on the situation when the coefficient of the odd terms $\epsilon=0$ and when adjacent elements are decoupled, $\gamma=0$ (the right-hand circle in Figure 1). Then centre manifold theory

\footnotetext{
${ }^{1}$ Natural symmetry also makes the general results most easy to express in terms of centred difference operators, and so I use them throughout this paper.

${ }^{2}$ The analysis presented here could be applied to modelling unstable dynamics, such as that from negative diffusion $u_{t}=-u_{x x}$. The difference is that the relevance theorem would no longer apply $-\mathcal{M}$ would not be attractive and the finite difference model would not capture the long term dynamics. The centre manifold model would, however, capture all the finite solutions, if any.
} 
[1, e.g.] guarantees the existence and relevance of a numerical model parametrised by the discrete values of the field at the grid points, $u_{j}$. The constructed model is accurate to the order of the residuals of the differential equation (1).

The spectrum of the linear dynamics is used to show there exists a centre manifold. Adjoin to (1) the trivial dynamical equations

$$
\dot{\gamma}=\dot{\epsilon}=0,
$$

so that terms multiplied by $\epsilon$ or $\gamma$ become "nonlinear terms" in the asymptotic expansion we develop about $\gamma=\epsilon=0$. Setting $\epsilon=0$ eliminates the odd terms to leave simply the linear equation $u_{t}=\mathcal{A} u$; for example, the diffusion equation $u_{t}=u_{x x}$. This is to be solved in the vicinity of $x_{j}$ for a field $u=e^{\lambda t} w(x)$, where here the dependence upon $j$ is implicit in the eigenfunction $w$ of $\mathcal{A} w=\lambda w$. This is a constant coefficient ODE and so has trigonometric general solutions $w \propto e^{i \alpha x}$ with corresponding eigenvalue $\lambda(\alpha)$ which is negative for nonzero wavenumber $\alpha$, as $\mathcal{A}$ is presumed generally dissipative; for example, $\lambda=-\alpha^{2}$ for the diffusion equation. The appropriate boundary conditions come from the nonlocal decoupling conditions that $w\left(x_{j \pm 1}\right)=w\left(x_{j}\right)$ from (3) with $\gamma=0$. Thus within each element the eigenmodes are: $\exp \left[i \alpha_{n}\left(x-x_{j}\right)\right]$ for even integers $n$, where $\alpha_{n}=n \pi / h$; and also $\sin \left[\alpha_{n}\left(x-x_{j}\right)\right]$ for odd integers $n$. The spectrum is then $\lambda_{n}=\lambda(n \pi / h)$; for example, $\lambda_{n}=-n^{2} \pi^{2} / h^{2}$ for the diffusion equation. Consequently, in the absence of inter-element coupling, generally expect all modes to decay exponentially quickly to zero on a time scale $\mathcal{O}\left(h^{2}\right)$ as $\lambda(\alpha)$ will be symmetric, except for the neutral mode, $n=0$, which is constant in $x, v_{j}(x, t)=u_{j}$. Thus for small enough $\epsilon$ and $\gamma$, theory ([2, p. 281] or [21, p. 96]) assures us that there exists a centre manifold $\mathcal{M}$ for the system (11) coupled across elements by (3). The centre manifold $\mathcal{M}$ is here, by (22), to be parametrised by the values of the field at the grid points, $u_{j}$. Thus using $\boldsymbol{u}$ to denote the set of grid values $u_{j}$, the "amplitudes", theory [1, 2, 21] supports our description of the centre manifold and the evolution thereon as

$$
u(x, t)=v(\boldsymbol{u}, x), \quad \text { such that } \dot{u}_{j}=g_{j}(\boldsymbol{u})
$$

(translational invariance in $x$ leads to identical expressions in each element except for appropriate changes of the subscript $j$ ). The evolution $\dot{u}_{j}=g_{j}(\boldsymbol{u})$ forms the holistic finite difference model. When the model is constructed to errors $\mathcal{O}\left(\gamma^{\ell}\right)$, then we account for interactions among $\ell-1$ elements on either side of any given element, and so the resulting finite difference model has a stencil of width $2 \ell-1$ on the spatial grid. I call these models "holistic" because, unlike traditional finite difference modelling which just analyses separately each term in the equations, here $\mathcal{M}$ is made up of actual solutions of the PDE and so here the discretisation models all the possible interactions between all the terms in the equations [18, 11, e.g.].

Moreover, the evolution on the centre manifold forms an accurate low-dimensional model of the dynamics of the PDE (11). Again provided $\epsilon$ and $\gamma$ are small enough, theory, [2, p. 282] or [21, p. 128], assures us that all solutions sufficiently near the centre manifold $\mathcal{M}$ are not only attracted to $\mathcal{M}$ but exponentially quickly approach the actual solutions of the PDE that make up $\mathcal{M}$. (The rate of attraction is approximated for practical purposes by the leading negative eigenvalue; for example, $\lambda_{1}=-\pi^{2} / h^{2}$ for the diffusion equation.) In the development of inertial manifolds by Temam [20] and others, this property is sometimes called the asymptotic completeness of the model, for example see Robinson [19] or Constantin et al. [3, $§ 12-3]$, and sometimes as exponential tracking [7] e.g.]. Observe that this is one 
of the crucial new aspects brought to finite difference modelling by centre manifold theory. It asserts that on a finite grid spacing we will faithfully track the solutions of the original PDE. There will be some limitations: steep gradients and large nonlinearities will test the model as always. But the centre manifold theory, as seen here in $\$ 3$ and in introductory work [18, 11, provides a rationale and a method to construct the requisite adjustments to a finite difference model to robustly model a wide range of dynamics on a finite grid spacing. The main limitation is the rate of attraction to $\mathcal{M}$ : the centre manifold model should be able to capture any dynamics occurring on a time-scale longer than $1 /\left|\lambda_{1}\right|\left(h^{2} / \pi^{2}\right.$ for diffusion). Thus the model evolution on $\mathcal{M},(7)$, captures all the long term dynamics with some provisos.

\section{Advection-Diffusion IS MODELlED ROBUSTly}

Here we explore perhaps the simplest nontrivial example in the class of PDE's: the advection-diffusion equation

$$
u_{t}=-\epsilon u_{x}+u_{x x},
$$

where $\epsilon$ is the advection speed; in other sections I treat $\epsilon$ as small but not in this section. This PDE fits into the scheme outlined in the previous sections with the operators $\mathcal{A}=\partial_{x}^{2}$ and $\mathcal{B}=-\partial_{x}$. We show consistency for small grid spacing $h$, and find interesting and stable upwind approximations for large $\epsilon h$. The associated sophisticated dependence upon $\epsilon$ is perhaps indicative of the need to treat odd operators differently from even.

Following the framework discussed in the previous section, seek a centre manifold and the evolution thereon, equation (7), in a power series in the inter-element coupling parameter $\gamma$ :

$$
u(x, t)=v_{j}=\sum_{k=0}^{\infty} \gamma^{k} v_{j}^{k}(\boldsymbol{u}, x), \quad \text { s.t. } \quad \dot{u}_{j}=g_{j}=\sum_{k=1}^{\infty} \gamma^{k} g_{j}^{k}(\boldsymbol{u}),
$$

where the superscripts on $v_{j}$ and $g_{j}$ are an index and do not denote exponentiation. Substitute (9) into the advection-diffusion equation (8) and equate like powers of $\gamma$ to determine

$$
\sum_{i} \sum_{l=1}^{k} g_{i}^{l} \frac{\partial v_{j}^{k-l}}{\partial u_{i}}=-\epsilon \frac{\partial v_{j}^{k}}{\partial x}+\frac{\partial^{2} v_{j}^{k}}{\partial x^{2}}, \quad k=0,1,2, \ldots
$$

Similarly, substituting (9) into the amplitude condition (2) and equating powers of $\gamma$ requires

$$
v_{j}^{0}\left(\boldsymbol{u}, x_{j}\right)=u_{j}, \quad \text { and } \quad v_{j}^{k}\left(\boldsymbol{u}, x_{j}\right)=0 \quad \text { for } 1 \leq k<\ell,
$$

whereas substituting (9) into the IBC (5) requires, evaluating the left-hand sides at $x_{j}$,

$$
\mu_{x} \delta_{x} v_{j}^{k}=\left\{\begin{array}{cc}
\mu \delta u_{j}, & k=1 \\
0, & k \neq 1
\end{array}\right.
$$

and

$$
\delta_{x}^{2} v_{j}^{k}=\left\{\begin{array}{cc}
\delta^{2} u_{j}, & k=1 \\
0, & k \neq 1
\end{array}\right.
$$

We proceed to solve the first few steps in this hierarchy of equations and interpret the resultant hierarchy of finite difference approximations. 
First explore the holistic finite difference model with only first-order interactions between adjacent elements, that is, solve for $0 \leq k<\ell=2$ so that errors are $\mathcal{O}\left(\gamma^{2}\right)$. In polynomials, the $k=0$ equations of (10) 12) have solution

$$
v_{j}^{0}=u_{j} .
$$

The solution of the $k=1$ equations give

$$
v_{j}^{1}=\left[\frac{e^{\epsilon x}-1}{4 \sinh ^{2}(\epsilon h / 2)}-\frac{\cosh (\epsilon h / 2)}{2 h \sinh (\epsilon h / 2)} x\right] \delta^{2} u_{j}+x \frac{\mu \delta}{h} u_{j}
$$

and

$$
g_{j}^{1}=-\frac{\mu \delta}{h} u_{j}+\nu_{1} \frac{\delta^{2}}{h^{2}} u_{j}
$$

where

$$
\nu_{1}=\frac{\epsilon h \cosh (\epsilon h / 2)}{2 \sinh (\epsilon h / 2)}
$$

is plotted in Figure 3. Substitute $\gamma=1$ to obtain the finite difference model for the advection-diffusion PDE (8):

$$
\dot{u}_{j}=-\epsilon \frac{\mu \delta}{h} u_{j}+\nu_{1} \frac{\delta^{2}}{h^{2}} u_{j}
$$

as $h \rightarrow 0$ this is equivalent to

$$
u_{t}=-\epsilon u_{x}+u_{x x}+\frac{h^{2}}{12}\left(\epsilon-\partial_{x}\right)^{2} u_{x x}+\mathcal{O}\left(h^{4}\right),
$$

and so is indeed consistent to $\mathcal{O}\left(h^{2}\right)$, independent of $\epsilon$, with the advection-diffusion PDE (8). Introduced automatically in this analysis is the novel enhancement of the dissipation term $\delta^{2} u_{j}$ by the factor $\nu_{1}$ which grows from 1 with $\epsilon h$. Alternatively, for small $\epsilon h$ from (17) $\nu_{1}-1 \approx \frac{\epsilon^{2}}{12} \delta^{2} u_{j}$ involves the square of the advection speed; thus one may also view this term as an upwind correction to the finite difference approximation of the first derivative:

$$
-\frac{\epsilon}{h} \mu \delta+\frac{\epsilon^{2}}{12} \delta^{2}=-\frac{\epsilon}{h}\left[\left(\frac{1}{2}-\frac{\epsilon h}{12}\right) E^{1 / 2}+\left(\frac{1}{2}+\frac{\epsilon h}{12}\right) E^{-1 / 2}\right] \delta,
$$

which increases the weight of the upwind grid point ( $E^{-1 / 2}$ is upwind if $\epsilon$ is positive). Either interpretation, as enhanced dissipation or upwind correction, is well known to be stabilising for finite advection speeds $\epsilon$.

It is interesting to explore the large $\epsilon h$ limit when there is strong advection across each element. From (17) $\nu_{1} \sim \epsilon h / 2$ as $\epsilon h \rightarrow \infty$ and is indeed within a few percent of this value for $\epsilon h>4$, see Figure 3. Thus for large advection speed $\epsilon$ on a finite width grid, the centre manifold analysis promotes the mode 3 (written in terms of the backward difference operator $\nabla$ )

$$
\dot{u}_{j} \approx-\frac{\epsilon}{h} \nabla u_{j}=-\frac{\epsilon}{h}\left(u_{j}-u_{j-1}\right) .
$$

This is not, and need not be, consistent with the PDE (8) as $h \rightarrow 0$, because it applies for finite $\epsilon h$. That it should be relevant to (8) comes from the centre manifold expansion in $\gamma$, albeit evaluated at $\gamma=1$ (via the "holistic" arrows in

\footnotetext{
${ }^{3}$ If the advection velocity is negative, $\epsilon<0$, then various signs change and the large $\epsilon h$ model remains an upwind model, but is consequently written in terms of forward differences. This also occurs for the later model (25), accurate to errors $\mathcal{O}\left(\gamma^{3}\right)$.
} 


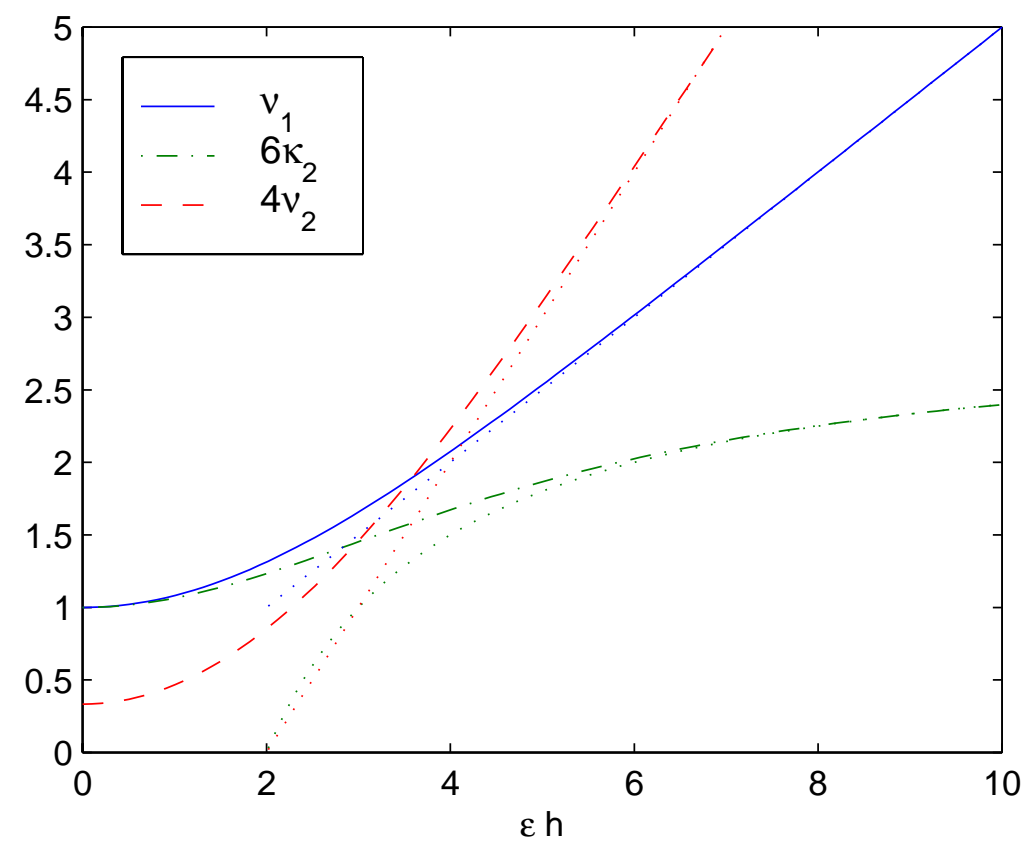

Figure 3. Coefficients of the centre manifold models (18) and (23) as a function of advection speed and grid spacing, $\epsilon h$. The dotted lines are the large $\epsilon h$ asymptotes: $\nu_{1} \approx \frac{\epsilon h}{2} ; \nu_{2} \approx \frac{\epsilon h}{4}-\frac{1}{2} ; \kappa_{2} \approx$ $\frac{1}{2}-\frac{1}{\epsilon h}$.

Figure 11. Exact solutions of (20) are readily obtained. For example, consider a point release from $j=0$ at $t=0: u_{j}(0)=1$ if $j=0$ and is 0 otherwise. Then the moment generating function $G(z, t)=\sum_{j=0}^{\infty} z^{j} u_{j}(t)$ is seen to be that for a Poisson probability distribution with parameter $\epsilon t / h$, namely $G(z, t)=\exp [(z-1) \epsilon t / h]$. Hence the mean location and variance of $u_{j}$ are

$$
\mu_{j}=\sigma_{j}^{2}=\frac{\epsilon t}{h} \Rightarrow \mu_{x}=\epsilon t \quad \text { and } \quad \sigma_{x}^{2}=\epsilon h t .
$$

Thus for $\epsilon h$ not small: this model has precisely the correct advection speed $\epsilon$; and although the variance is quantitatively wrong, $\epsilon h t$ instead of $2 t$, at least it is qualitatively correct for finite $\epsilon h$. The centre manifold model (18) is consistent for small $h$ and has the virtue of being always stable, and will always maintain nonnegativity of solutions no matter how large the advection speed $\epsilon$.

Second, explore the holistic finite difference model with second-order interactions between adjacent elements, that is, solve for $0 \leq k<\ell=3$ so that errors are $\mathcal{O}\left(\gamma^{3}\right)$. The details of $v_{j}^{2}$ are of little direct interest here. The finite difference model depends directly upon

$$
g_{j}^{2}=+\frac{\epsilon}{h} \kappa_{2} \mu \delta^{3} u_{j}+\frac{1}{h^{2}}\left(\delta^{2}-\nu_{1} \delta^{2}-\nu_{2} \delta^{4}\right) u_{j},
$$

where

$$
\nu_{2}=\frac{\epsilon h \cosh (\epsilon h / 2)}{4 \sinh (\epsilon h / 2)}-\frac{1}{2}+\frac{\epsilon h \cosh (\epsilon h / 2)}{8 \sinh ^{3}(\epsilon h / 2)}-\frac{1}{4 \sinh ^{2}(\epsilon h / 2)}
$$


and

$$
\kappa_{2}=\frac{1}{2}+\frac{1}{2 \sinh ^{2}(\epsilon h / 2)}-\frac{\cosh (\epsilon h / 2)}{\epsilon h \sinh (\epsilon h / 2)} .
$$

Observe the marvellous feature that when we form $\gamma g_{j}^{1}+\gamma^{2} g_{j}^{2}$ evaluated at $\gamma=1$ the terms in $\nu_{1}(\epsilon h) \delta^{2}$ neatly cancel. The model for the advection-diffusion thus reduces to

$$
\dot{u}_{j}=-\frac{\epsilon}{h}\left(\mu \delta-\kappa_{2} \mu \delta^{3}\right) u_{j}+\frac{1}{h^{2}}\left(\delta^{2}-\nu_{2} \delta^{4}\right) u_{j} .
$$

We see in this model that, as $h \rightarrow 0$, the hyperdiffusion coefficient $\nu_{2} \sim 1 / 12$ and the dispersion coefficient $\kappa_{2} \sim 1 / 6$, to give the classic second-order in $h$ corrections to the central difference approximations of the first two derivatives. Indeed, as $h \rightarrow 0$ the model (23) is equivalent to

$$
u_{t}=-\epsilon u_{x}+u_{x x}+\frac{h^{4}}{90}\left(\epsilon-\partial_{x}\right)^{3} u_{x x x}+\mathcal{O}\left(h^{6}\right),
$$

and so is consistent to $\mathcal{O}\left(h^{4}\right)$, independent of $\epsilon$, with the advection-diffusion PDE (8).

For large advection speed or grid size, large $\epsilon h$, the model (23) is astonishingly good. Using the large $\epsilon h$ approximations plotted in Figure 3 for $\nu_{2}$ and $\kappa_{2}$, the model (23) reduces to simply

$$
\begin{aligned}
\dot{u}_{j} & =-\frac{\epsilon}{h}\left(\nabla+\frac{1}{2} \nabla^{2}\right) u_{j}+\frac{1}{h^{2}} \nabla^{2} u_{j} \\
& =-\frac{\epsilon}{2 h}\left(u_{j-2}-4 u_{j-1}+3 u_{j}\right)+\frac{1}{h^{2}}\left(u_{j-2}-2 u_{j-1}+u_{j}\right) .
\end{aligned}
$$

This large $\epsilon h$ model uses only backward differences to incorporate second-order upwind estimates of the derivative, $\nabla+\frac{1}{2} \nabla^{2}$, and the second derivative, $\nabla^{2}$. To show its good properties 4 consider again a point release from $j=0$ at time $t=0$. The moment generating function $G(z, t)=\sum_{j=0}^{\infty} z^{j} u_{j}(t)$ for the evolution governed by (25) is readily shown to be

$$
G(z, t)=\exp \left[-\frac{\epsilon t}{2 h}(z-1)(z-3)+\frac{t}{h^{2}}(z-1)^{2}\right] .
$$

Then, since

$$
\left.\frac{\partial G}{\partial z}\right|_{z=1}=\frac{\epsilon t}{h} \quad \text { and }\left.\quad \frac{\partial^{2} G}{\partial z^{2}}\right|_{z=1}=\left(\frac{\epsilon t}{h}\right)^{2}-\frac{\epsilon t}{h}+\frac{2 t}{h^{2}},
$$

we determine the mean position and variance of the spread in $u_{j}$ to be

$$
\mu_{j}=\frac{\epsilon t}{h} \text { and } \sigma_{j}^{2}=\frac{2 t}{h^{2}} \Rightarrow \mu_{x}=\epsilon t \text { and } \sigma_{x}^{2}=2 t .
$$

This predicted mean and variance following a point release are exactly correct for all time for the advection-diffusion PDE (8). This specific result applies to all finite advection speeds $\epsilon$ and all finite grid spacings $h$ whenever $\epsilon h$ is large enough.

It seems that creating finite differences which, as shown in Figure 1, are both consistent for small grid spacing $h$ and also holistically derived via centre manifold

\footnotetext{
${ }^{4}$ The upwind difference model 25 is only stable for $\epsilon h>2 / 3$. However, from Figure 3 the approximation 25] is only applicable to 23] for $\epsilon$ greater than about 4; thus its instability for very much smaller $\epsilon h$ is irrelevant.
} 
theory thus can lead to models which are remarkably accurate and stable over a wide range of parameters.

\section{Models of the even terms are Consistent}

In this section I prove that the proposed centre manifold approach consistently models all the even derivatives in $\mathcal{A}$. That is, the equivalent PDE of the finite difference model on the centre manifold $\mathcal{M}$ matches $u_{t}=\mathcal{A} u$ to some order in grid spacing $h$; the order of error is controlled by the order of truncation, $\ell$, in the coupling coefficient $\gamma$. The veracity of the following theorems is supported by the results of suitable variants of a computer algebra program available from the author.

Remarkably, the polynomials found here to describe the structure within each element are independent of the linear operator $\mathcal{A}$ !

Theorem 1. The centre manifold model (7), constructed with the amplitude condition (2), the internal boundary condition (3) and to errors $\mathcal{O}\left(\gamma^{\ell}\right)$, forms a semidiscrete finite difference approximation to the PDE $u_{t}=\mathcal{A} u$ consistent to $\mathcal{O}\left(\partial_{x}^{2 \ell}\right)$, where $\mathcal{A}$ is any even operator.

Proof. I construct the proof in stages beginning with the end result and finding successive sufficient conditions for the preceding steps. Observe that I actually prove a slightly stronger result: by allowing the even operator $\mathcal{A}$ to contain a constant term $a_{0}$, see (32), I prove the consistency of an invariant manifold model based upon the mode with eigenvalue $\lambda_{0}=a_{0}$. When $a_{0} \geq 0$ this forms a centreunstable manifold model for which a relevance theorem also ensures asymptotic completeness.

- To solve $u_{t}=\mathcal{A} u$ to errors $\mathcal{O}\left(\gamma^{\ell}\right)$, expand the centre manifold model (7) to $\mathcal{O}\left(\gamma^{\ell}\right)$ as

$$
u=v(\boldsymbol{u}, x)=\sum_{k=0}^{\ell-1} \gamma^{k} v_{j}^{k}(\boldsymbol{u}, x), \quad \text { and } \quad \dot{u}_{j}=g(\boldsymbol{u})=\sum_{k=0}^{\ell-1} \gamma^{k} g^{k} u_{j},
$$

where hereafter $g^{k}$ are some finite difference operators (as before, superscripts to $\gamma$ denote exponentiation whereas those on $v$ and $g$ denote the index of coefficients in the asymptotic expansion). Then substitution into the PDE and extracting powers of $\gamma$ shows that we require

$$
\mathcal{A} v_{j}^{k}=g^{0} v_{j}^{k}+g^{1} v_{j}^{k-1}+\cdots+g^{k-1} v_{j}^{1}+g^{k} v_{j}^{0} \quad \text { for } 0 \leq k<\ell .
$$

Similarly we require the amplitude condition (21) and the IBC (13). Equations (29) and (11) 13) form a well-posed system of equations for $v_{j}^{k}$ and $g^{k}$. In many applications of centre manifold theory, because $\mathcal{A}-g^{0}$ is singular, we often solve each level in the hierarchy of equations in two steps: the first is to find $g^{k}$ by ensuring that the right-hand side of (29) is in the range of $\mathcal{A}-g^{0}$ (this is the so-called "solvability condition"); the second step is to find $v_{j}^{k}$. However, here we proceed to construct straightforwardly the solution of the entire set of equations in general.

- We show the hierarchy of differential equations (29) are satisfied by functions $v_{j}^{k}$ and give consistent finite difference operators $g^{k}$, if $v_{j}^{k}$ satisfy the 
recursive difference equation

$$
\delta_{x}^{2} v_{j}^{k}=\delta^{2} v_{j}^{k-1} \quad \text { for all } x, \text { and } \quad v_{j}^{0}=\text { constant } .
$$

- By the following induction argument (30) implies that

$$
\delta_{x}^{2 m} v_{j}^{k}= \begin{cases}\delta^{2 m} v_{j}^{k-m}, & \text { for } m=0, \ldots, k \\ 0, & m=k+1, k+2, \ldots\end{cases}
$$

Now,

$$
\begin{aligned}
\delta_{x}^{2 m} v_{j}^{k} & =\delta_{x}^{2 m-2} \delta_{x}^{2} v_{j}^{k} \\
& =\delta_{x}^{2 m-2} \delta^{2} v_{j}^{k-1} \quad \text { by (30) } \\
& =\delta^{2} \delta_{x}^{2(m-1)} v_{j}^{k-1} \quad \text { as } \delta \text { and } \delta_{x} \text { commute } \\
& =\delta^{2} \delta^{2(m-1)} v_{j}^{k-m} \quad \text { p.v. (31) holds for } m-1 \\
& =\delta^{2 m} v_{j}^{k-m} .
\end{aligned}
$$

Then since (31) is trivially true for $m=0$, it follows by induction that (31) holds for all $m \leq k$. Further, since $v_{j}^{0}$ is constant by (30), $\delta_{x}^{2 k} v_{j}^{k}$ is constant, so higher order differences $(m>k)$ are all zero.

- By an "even" operator $\mathcal{A}$ I mean one which only involves even order derivatives in $x$. Hence write $\mathcal{A}$ formally as an infinite sum of even powers of the central difference operator

$$
\mathcal{A}=\sum_{m=0}^{\infty} a_{m} \delta_{x}^{2 m}
$$

for some coefficients $a_{m}$; for example, for the diffusion operator in (8), from [13, p. 65 , e.g.],

$$
\frac{\partial^{2}}{\partial x^{2}}=\frac{4}{h^{2}} \operatorname{arcsinh}^{2}\left(\frac{1}{2} \delta_{x}\right)=\frac{1}{h^{2}} \delta_{x}^{2}-\frac{1}{12 h^{2}} \delta_{x}^{4}+\frac{1}{90 h^{2}} \delta_{x}^{6}-\cdots ;
$$

more generally, $\mathcal{A}$ could be any symmetric convolution operator for which the infinite sum (32) forms a reasonable representation. Then (31) ensures (29), since

$$
\begin{aligned}
\mathcal{A} v_{j}^{k} & =\sum_{m=0}^{\infty} a_{m} \delta_{x}^{2 m} v_{j}^{k} \\
& =\sum_{m=0}^{k} a_{m} \delta^{2 m} v_{j}^{k-m} \text { by (31) } \\
& =g^{0} v_{j}^{k}+g^{1} v_{j}^{k-1}+\cdots+g^{k-1} v_{j}^{1}+g^{k} v_{j}^{0},
\end{aligned}
$$

provided $g^{k}=a_{k} \delta^{2 k}$, which are precisely the operators required to make the model $\dot{u}_{j}=g(\boldsymbol{u})$ of $u_{t}=\mathcal{A} u$ consistent to $\mathcal{O}\left(\partial_{x}^{2 \ell}\right)$, when truncated as in (28) to errors $\mathcal{O}\left(\gamma^{\ell}\right)$.

- By simple substitution, a sequence of functions $v_{j}^{k}$ satisfying the recurrence (30), amplitude conditions (11) and the internal boundary conditions (13) are

$$
v_{j}^{0}=u_{j}, \quad v_{j}^{k}=p_{k}(\xi) \mu \delta^{2 k-1} u_{j}+q_{k}(\xi) \delta^{2 k} u_{j}, \quad \text { for } k \geq 1,
$$



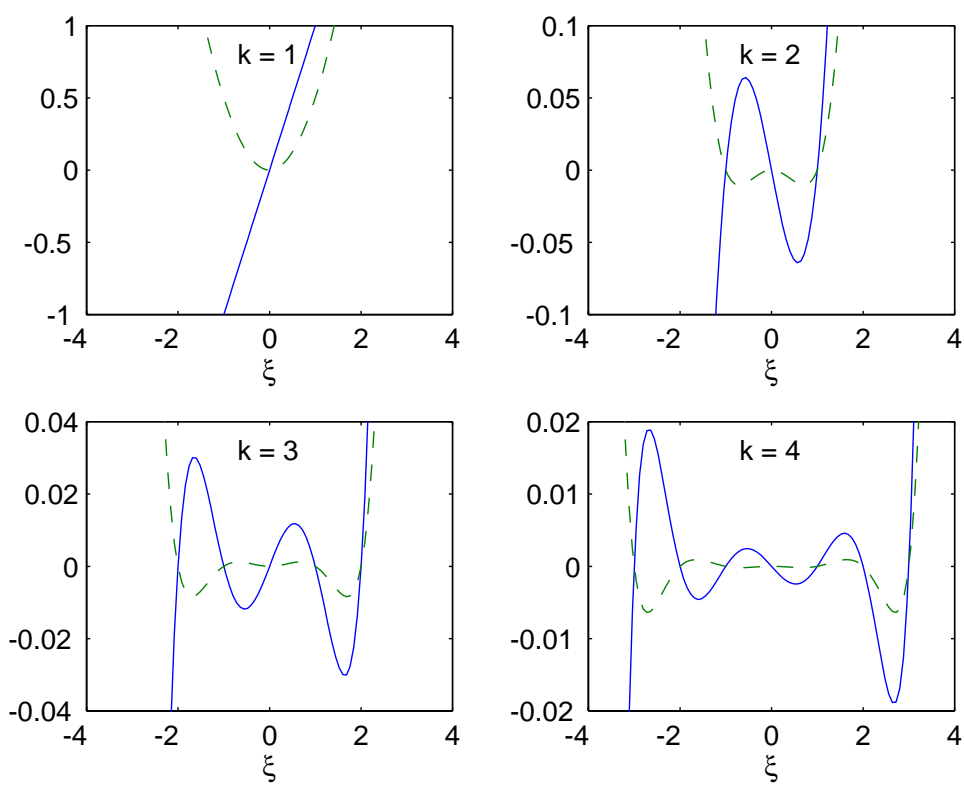

Figure 4. Graphs of the polynomials $p_{k}(\xi)$ (solid) and $q_{k}(\xi)$ (dashed), see (36), forming a basis for the fields of the approximations to the centre manifold.

where, as always, $\xi=\left(x-x_{j}\right) / h$ is a grid scaled coordinate, and provided that for $k \geq 1$

$$
\delta_{x}^{2} p_{k}=p_{k-1}, \quad p_{k}( \pm k)= \pm 1, \quad \text { and } \quad p_{k}(\xi)=0 \text { for } \xi=0, \pm 1,
$$

and similarly

$$
\delta_{x}^{2} q_{k}=q_{k-1}, \quad q_{k}( \pm k)=+\frac{1}{2}, \quad \text { and } \quad q_{k}(\xi)=0 \text { for } \xi=0, \pm 1,
$$

after defining $q_{0}(x)=1$ and $p_{0}(x)=0$.

- Analysing the difference tables for $p_{k}$ and $q_{k}$ and straightforward induction using (34 35) proves that $p_{k}(\xi)=q_{k}(\xi)=0$ for integers $\xi \in[-k+1, k-1]$. Then the following $p_{k}$ and $q_{k}$ are the unique polynomials, of degree $2 k-1$ and $2 k$ respectively, also satisfying $p_{k}( \pm k)= \pm 1$ and $q_{k}( \pm k)=+\frac{1}{2}$ :

$$
p_{k}(\xi)=\frac{1}{(2 k-1) !} \prod_{m=-k+1}^{k-1}(\xi-m), \quad \text { and } \quad q_{k}(\xi)=\frac{\xi}{2 k} p_{k}(\xi),
$$

as plotted in Figure 4. For example, $p_{1}(\xi)=\xi$ and $q_{1}(\xi)=\frac{1}{2} \xi^{2}$.

These polynomials $p_{k}$ and $q_{k}$ also need to satisfy the recurrences in (34) 35) pointwise in $\xi$. This is trivially true for $k=1$. Now, $\delta_{x}^{2} p_{k+1}$ is from (36) a polynomial of degree $2 k-1$, from its difference table has the same zeros as $p_{k}$, it is \pm 1 at $\pm k$, and so must be $p_{k}(\xi)$ for all $\xi$. Similarly for $\delta_{x}^{2} q_{k+1} 5$

\footnotetext{
${ }^{5}$ One easily imagines other functions $p_{k}$ and $q_{k}$ that have all the requisite properties to ensure a consistent finite difference approximation $g(\boldsymbol{u})$. For example, $\tilde{p}_{k}(\xi)=p_{k}(\xi)+a \sin (2 n \pi \xi)$. However, as is often the case, if the method of construction of the centre manifold makes $v_{j}^{k}$ a polynomial of degree $2 k$, then the solution for $v_{j}^{k}$ is the one given in (33) in terms of $p_{k}$ and $q_{k}$.
} 
Corollary 2. It follows immediately from the theorem that if the highest derivative in the even operator $\mathcal{A}$ is of order $n$, then the finite difference model (28) is consistent with $u_{t}=\mathcal{A} u$ to $\mathcal{O}\left(h^{2 \ell-n}\right)$ as $h \rightarrow 0$.

\section{OdD PERTURBATIONS ARE ALSO CONSISTENT}

The results of the previous section on the modelling of even operators $\mathcal{A}$ are extremely satisfactory. The modelling of odd operators is not quite so neat. In the general linear PDE (1) I introduced the odd terms, $\mathcal{B}$, with a multiplying $\epsilon$. The reason is, as seen in $\$ 3$, that such odd terms generate extra terms in the finite difference model which are nonlinear in the coefficients of the odd derivatives, that is, $\mathcal{O}\left(\epsilon^{2}\right)$. As elaborated in $\$ 3$ for the advection-diffusion equation, these higher-order contributions seem to reflect the changes needed for stable discretisations of equations with large amounts of advection, $u_{x}$, or dispersion, $u_{x x x}$. The extra complications of these nontrivial effects of odd derivatives appear necessary. However, here we restrict attention to proving consistency to an error quadratic in the odd coefficients and leave to further research the investigation of higher-order consistency.

Theorem 3. The centre manifold model (7), constructed with the amplitude condition (2), the internal boundary condition (3) and to errors $\mathcal{O}\left(\gamma^{\ell}, \epsilon^{2}\right)$, forms a finite difference approximation to the PDE $u_{t}=\mathcal{A} u+\epsilon \mathcal{B} u$ consistent to $\mathcal{O}\left(\partial_{x}^{2 \ell}+\epsilon \partial_{x}^{2 \ell-1}, \epsilon^{2}\right)$, where $\mathcal{A}$ is any even operator and $\mathcal{B}$ is any odd operator.

Proof. As in (28), we expand the the centre manifold ansatz (17) to errors $\mathcal{O}\left(\gamma^{\ell}, \epsilon^{2}\right)$ :

$$
u=\sum_{k=0}^{\ell-1} \gamma^{k} v_{j}^{k}+\epsilon \sum_{k=0}^{\ell-1} \gamma^{k} w_{j}^{k}, \quad \text { and } \quad \dot{u}_{j}=\sum_{k=0}^{\ell-1} \gamma^{k} g^{k} u_{j}+\epsilon \sum_{k=0}^{\ell-1} \gamma^{k} f^{k} u_{j},
$$

where $f^{k}$ and $g^{k}$ are difference operators (as before the superscript to $v, w, g$ and $h$ denotes an index in the series, whereas the superscript to $\gamma$ denotes exponentiation). After substitution into the PDE, terms in $\epsilon^{0}$ determine $v_{j}^{k}$ and $g^{k}$ as in the previous section. Upon extracting from the PDE the coefficients of terms linear in $\epsilon$ and of various powers of $\gamma$, we are required to solve

$$
\mathcal{A} w_{j}^{k}+\mathcal{B} v_{j}^{k}=\sum_{r=0}^{k}\left(f^{k-r} v_{j}^{r}+g^{k-r} w_{j}^{r}\right), \quad \text { for } 0 \leq k<\ell .
$$

Substitution of the expansion (37) into the amplitude condition (21) and the IBC's (5) and equating coefficients of $\epsilon \gamma^{k}$ leads to these internal boundary conditions for the $w_{j}^{k}(x)$ :

$$
w_{j}^{k}=0 \text { for } x=x_{j}, x_{j \pm 1} .
$$

Since $\mathcal{B}$ is an odd operator, we formally write it as the following infinite sum of odd powers of centred difference operators:

$$
\mathcal{B}=\sum_{m=1}^{\infty} b_{m} \mu_{x} \delta_{x}^{2 m-1},
$$

for some coefficients $b_{m}$; for example, from [13 p. 65 , e.g.],

$$
\frac{\partial}{\partial x}=\frac{2 \mu_{x}}{h} \operatorname{arcsinh}\left(\frac{1}{2} \delta_{x}\right)=\frac{1}{h} \mu_{x} \delta_{x}-\frac{1}{6 h} \mu_{x} \delta_{x}^{3}+\frac{1}{30 h} \mu_{x} \delta_{x}^{5}-\cdots ;
$$


and more generally, $\mathcal{B}$ could be any antisymmetric convolution operator for which the infinite sum (40) is reasonable. I prove that there exist solutions $w_{j}^{k}(x)$, odd functions of $x$ (about $x_{j}$ ), with

$$
f^{k}=b_{k} \mu \delta^{2 k-1},
$$

so that the model (37) is consistent with the effect of the odd derivatives in $\epsilon \mathcal{B}$ to errors $\mathcal{O}\left(\delta_{x}^{2 \ell-1}\right)=\mathcal{O}\left(\partial_{x}^{2 \ell-1}\right)$. Since we already know $v_{j}^{k}$ and since $w_{j}^{k}$ appears to vary for different problems, the operators $f^{k}$ are determined by the solvability condition that all terms except $\mathcal{A} w_{j}^{k}-g^{0} w_{j}^{k}$ appearing in (38) combine to be in the range of the singular $\mathcal{A}-g^{0}$.

- First, prove the even part of $\mathcal{B} v_{j}^{k}$ cancels with the even part of $\sum_{r=0}^{k} f^{k-r} v_{j}^{r}$ and so is eliminated from (38). As a preliminary step consider, using (36),

$$
\begin{aligned}
\mu_{x} \delta_{x} p_{k}(\xi) & =\frac{1}{(2 k-1) !} \frac{1}{2}\left[\prod_{m=-k+1}^{k-1}(\xi+1-m)-\prod_{m=-k+1}^{k-1}(\xi-1-m)\right] \\
& =\frac{1}{(2 k-1) !} \prod_{m=-k+2}^{k-2}(\xi-m) \\
& \times \frac{1}{2}[(\xi+k)(\xi+k-1)-(\xi-k)(\xi-k+1)] \\
= & \frac{1}{(2 k-1)(2 k-2)} p_{k-1}(\xi) \times(2 k-1) \xi \\
= & q_{k-1}(\xi) .
\end{aligned}
$$

Then from (33) and since $p_{k}$ is odd and $q_{k}$ is even, see Figure $4, \mathcal{B} q_{k}$ is odd, and so the even part of $\mathcal{B} v_{j}^{k}$ is

$$
\begin{aligned}
\mathcal{B} p_{k} \mu \delta^{2 k-1} u_{j} & =\sum_{m=1}^{\infty} b_{m} \mu_{x} \delta_{x}^{2 m-1} p_{k} \mu \delta^{2 k-1} u_{j} \quad \text { by (40) } \\
& =\sum_{m=1}^{k} b_{m} \mu_{x} \delta_{x} p_{k-m+1} \mu \delta^{2 k-1} u_{j} \quad \text { by (35) inductively } \\
& =\sum_{m=1}^{k} b_{m} q_{k-m} \mu \delta^{2 k-1} u_{j} \quad \text { by (42), }
\end{aligned}
$$

whereas on the right-hand side of (38) the even part of $\sum_{r=0}^{k} f^{k-r} v_{j}^{r}$ is

$$
\begin{aligned}
\sum_{r=0}^{k} f^{k-r} q_{r} \delta^{2 r} u_{j} & =\sum_{r=0}^{k} q_{r} b_{k-r} \mu \delta^{2(k-r)-1} \delta^{2 r} u_{j} \quad \text { by (41) } \\
& =\sum_{r=0}^{k} q_{r} b_{k-r} \mu \delta^{2 k-1} u_{j}
\end{aligned}
$$

which exactly cancels with (42) from the even part of $\mathcal{B} v_{j}^{k}$ on the left-hand side of 38.

- Second, since the even components of (38) that involve the various $v_{j}^{k}$ cancel, and since $\mathcal{A}$ and $g^{k}$ are even, then a particular solution $w_{j}^{k}(x)$ of (38) may 
be found that is odd. The conditions (B9) then force these odd $w_{j}^{k}$ to be the unique solutions in the space of finite degree polynomials.

- Third, consider evaluating the hierarchy of equations (38) at the centre grid point of the element, $x=x_{j}$ or equivalently $\xi=0$, and simplify the various contributions.

- Now $\mathcal{A}$ is an even operator and $w_{j}^{k}$ an odd function, so $\mathcal{A} w_{j}^{k}$ is an odd function, and thus $\mathcal{A} w_{j}^{k}\left(x_{j}\right)=0$.

- Since $g^{k-r}$ is a difference operator in $j$ it does not affect the amplitude condition that $w_{j}^{r}\left(x_{j}\right)=0$, and thus $g^{k-r} w_{j}^{r}\left(x_{j}\right)=0$.

- I have already shown that the even parts of $\mathcal{B} v_{j}^{k}$ and $\sum_{r=0}^{k} f^{k-r} v_{j}^{r}$ agree pointwise, so they certainly do at $x_{j}$, at which the odd parts must also trivially vanish together.

Thus the choice (41) is the unique one to satisfy this solvability condition for (38).

Since the expansion (37) then contains the exact differences up to $b_{\ell-1} \mu \delta^{2 \ell-3}$ and $a_{\ell-1} \delta^{2 \ell-2}$, the errors in the finite truncation of the finite difference model will be $\mathcal{O}\left(\partial_{x}^{2 \ell}\right)$ from the even terms and $\mathcal{O}\left(\epsilon \partial_{x}^{2 \ell-1}, \epsilon^{2}\right)$ from the odd.

Corollary 4. It follows immediately from the theorem that if the highest derivative in the operator $\mathcal{A}+\epsilon \mathcal{B}$ is of order $n$, then the finite difference model (37) is consistent with $u_{t}=\mathcal{A} u+\epsilon \mathcal{B} u$ to $\mathcal{O}\left(h^{2 \ell-1-n}\right)$ as $h \rightarrow 0$ to an error $\mathcal{O}\left(\epsilon^{2}\right)$.

\section{Concluding Remarks}

We have seen that the artificial internal boundary conditions (31) together with the application of centre manifold theory generate finite difference models that have remarkably good properties, at least for linear systems. These are explicitly shown for the example of the advection-diffusion equation (\$3i), where we saw not only consistency for small $h$, but also an appropriate upwind model for large $\epsilon h$. Although Theorem 3 only establishes consistency with the odd terms to $\mathcal{O}\left(\epsilon^{2}\right)$, the advection-diffusion example of 93 shows that higher-order consistency is possible. Further research is needed on the characteristics of higher orders in the odd derivatives.

Also, further research, such as that for Burgers' equation in [18], will explore the performance of this holistic approach to discretisations of nonlinear systems in various spatial dimensions. Since centre manifold theory is designed to analyse nonlinear systems, I expect reliable models to be derived.

Throughout the analysis in this paper I have parametrised the centre manifold model in terms of the field at each of the grid points, $u_{j}=u\left(x_{j}, t\right)$. This was done for simplicity. Other choices are possible for the parameters of the finite difference model, for example we could choose to use the element average

$$
u_{j}(t)=\frac{1}{h} \int_{x_{j}-h / 2}^{x_{j}+h / 2} u(x, t) d x .
$$

This choice would be appropriate to easily establish the conservation of total $u$, or not, as the case may be. Computational experiments show that either of these choices of amplitude produce equivalent results for linear systems.

Centre manifold theory is routinely applied to autonomous dynamical systems. However, the geometric viewpoint it establishes leads to a rational treatment of 
the projection of initial conditions onto the finite dimensional model, and of the projection of a perturbing forcing [17, 4, 14].

\section{ACKNOWLEDGMENT}

This research was supported by a grant from the Australian Research Council.

\section{REFERENCES}

[1] J. Carr. Applications of centre manifold theory, volume 35 of Applied Math. Sci. SpringerVerlag, 1981. MR 83g:34039

[2] J. Carr and R. G. Muncaster. The application of centre manifold theory to amplitude expansions. II. Infinite dimensional problems. J. Diff. Eqns, 50:280-288, 1983. MR 85d:58057b

[3] P. Constantin, C. Foias, B. Nicolaenko, and R. Temam. Integral manifolds and inertial manifolds for dissipative partial differential equations, volume 70 of Applied Math. Sci. SpringerVerlag, 1989. MR 90a:35026

[4] S. M. Cox and A. J. Roberts. Initial conditions for models of dynamical systems. Physica D, 85:126-141, 1995. MR 96e:34072

[5] C. Foias, M. S. Jolly, I. G. Kevrekedis, G. R. Sell, and E. S. Titi. On the computation of inertial manifolds. Phys Lett. A, 131:433-436, 1988.

[6] C. Foias, M. S. Jolly, I. G. Kevrekidis, and E. S. Titi. Dissipativity of numerical schemes. Nonlinearity, 4:591-613, 1991. MR 89k:65154

[7] C. Foias, G. R. Sell, and E. S. Titi. Exponential tracking and approximation of inertial manifolds for dissipative nonlinear equations. J. Dyn. \& Diff. Eqns, 1:199-244, 1989. MR 90k:35031

[8] C. Foias and E. S. Titi. Determining nodes, finite difference schemes and inertial manifolds. Nonlinearity, 4:135-153, 1991. MR 92a:65241

[9] B. García-Archilla and E. S. Titi. Postprocessing the Galerkin method: the finite element case. SIAM J. Num. Anal., 37:470-499, 2000. MR 2001h:65112

[10] M. S. Jolly, I. G. Kevrekidis, and E. S. Titi. Approximate inertial manifolds for the KuramotoSivashinsky equation: analysis and computations. Physica D, 44:38-60, 1990. MR 91f:35217

[11] T. Mackenzie and A. J. Roberts. Holistic finite differences accurately model the dynamics of the Kuramoto-Sivashinsky equation. ANZIAM J., 42(E):C918-C935, 2000. [Online] http://anziamj . austms.org.au/V42/CTAC99/Mack.

[12] M. Marion and R. Temam. Nonlinear Galerkin methods. SIAM J. Numer. Anal., 26(5):11391157, 1989. MR 91a:65220

[13] National Physical Laboratory. Modern Computing Methods, 2nd ed., volume 16 of Notes on Applied Science. Her Majesty's Stationary Office, 1961. MR 22:8637

[14] A. J. Roberts. Appropriate initial conditions for asymptotic descriptions of the long term evolution of dynamical systems. J. Austral. Math. Soc. B, 31:48-75, 1989. MR 90h:58075

[15] A. J. Roberts. The utility of an invariant manifold description of the evolution of a dynamical system. SIAM J. Math. Anal., 20:1447-1458, 1989. MR 91f:58088

[16] A. J. Roberts. Low-dimensional models of thin film fluid dynamics. Phys. Letts. A, 212:63-72, 1996. MR 97b:76041

[17] A. J. Roberts. Computer algebra derives correct initial conditions for low-dimensional dynamical models. Comput. Phys. Comm., 126(3):187-206, 2000.

[18] A. J. Roberts. Holistic discretisation ensures fidelity to Burgers' equation. Applied Numerical Math., 37:371-396, 2001.

[19] J. C. Robinson. The asymptotic completeness of inertial manifolds. Nonlinearity, 9:13251340, 1996. MR 98m:34124

[20] R. Temam. Inertial manifolds. Mathematical Intelligencer, 12:68-74, 1990. MR 91i:58087.

[21] A. Vanderbauwhede. Centre manifolds, normal forms and elementary bifurcations. Dynamics Reported, pages 89-169, 1989. MR 90g:58092

Department of Mathematics and Computing, University of Southern Queensland, Toowoomba, Queensland 4352, Australia

E-mail address: aroberts@usq.edu.au 